\title{
- OBSERVATIONS ON THE SEPARATION OF IRON ORE IN A PROTOTYPE BATCH JIG
}

\author{
N. Naudé ${ }^{a},{ }^{,}$, L. Lorenzen ${ }^{b}$, A.V. Kolesnikov ${ }^{c}$, C. Aldrich ${ }^{b}$ and L. Auret ${ }^{b}$ \\ ${ }^{a}$ Department of Material Science and Metallurgical Engineering, \\ University of Pretoria, Pretoria, South Africa \\ *E-mail: Natasia.naude@up.ac.za \\ ${ }^{b}$ Department of Processing Engineering, University of Stellenbosch, Private Bag X1, Matieland, 7602, \\ Stellenbosch, South Africa, \\ ${ }^{c}$ Department of Chemical and Metallurgical Engineering, Tshwane University of Technology, Pretoria, South \\ Africa
}

\begin{abstract}
The separation of iron ore in a prototype batch jig separator developed by Mintek in South Africa, referred to as the Mineral Density Separator (MDS), can separate ore samples into multiple bulk density fractions, provided that narrow feed size ranges are used. In this study, the performance of the equipment is considered by use of iron ore particles coloured according to density. This facilitated visualization of the density composition of the stratified ores. The highest density iron ore $\left(>4000 \mathrm{~kg} / \mathrm{m}^{3}\right)$ could be separated efficiently from gangue material, but satisfactory stratification of lower density particles $\left(<3600 \mathrm{~kg} / \mathrm{m}^{3}\right)$ could not be achieved.
\end{abstract}

Keywords: Gravity Concentration; Iron Ores

\section{Introduction}

The Sishen iron ore mine is located in the Northern Cape Province of South Africa, approximately $220 \mathrm{~km}$ northeast of Upington and $280 \mathrm{~km}$ northwest of Kimberley (Pretorius and Hoffmann, 2007). The current Sishen Mine is considered to be one of the largest open pit iron ore operations in the world, producing approximately 27 million tonnes of iron ore per annum.

Jigging technology plays an important role in the mine's continued quest for process improvement and has progressed to a point where Sishen's run of mine material, once considered as waste, can be beneficiated to produce a saleable $64 \%$ iron. Further improvement of the technology would directly translate to greater process efficiencies, but progress is hampered by an incomplete understanding of the underlying separation mechanisms and effects of operating variables on the process. As a consequence, Mintek in South Africa has developed and tested a prototype pneumatically operated batch jig with programmable logic controllers (PLCs), referred to as the Mineral Density Separator (MDS). 
The MDS portrayed schematically in Figure 1, can be used to separate heavy minerals into different bulk density classes and can serve as a means to evaluate and predict jigging plant efficiencies. In the MDS the cylindrical chamber could accommodate rings of different sizes, viz. rings with an inner diameter of $200 \mathrm{~mm}$ and $315 \mathrm{~mm}$. The MDS is capable of fractionating an ore sample into ten different density layers, and is able to handle ore with a wide variety of densities, while treating material within the size range of $0.5 \mathrm{~mm}$ up to 30 $\mathrm{mm}$.

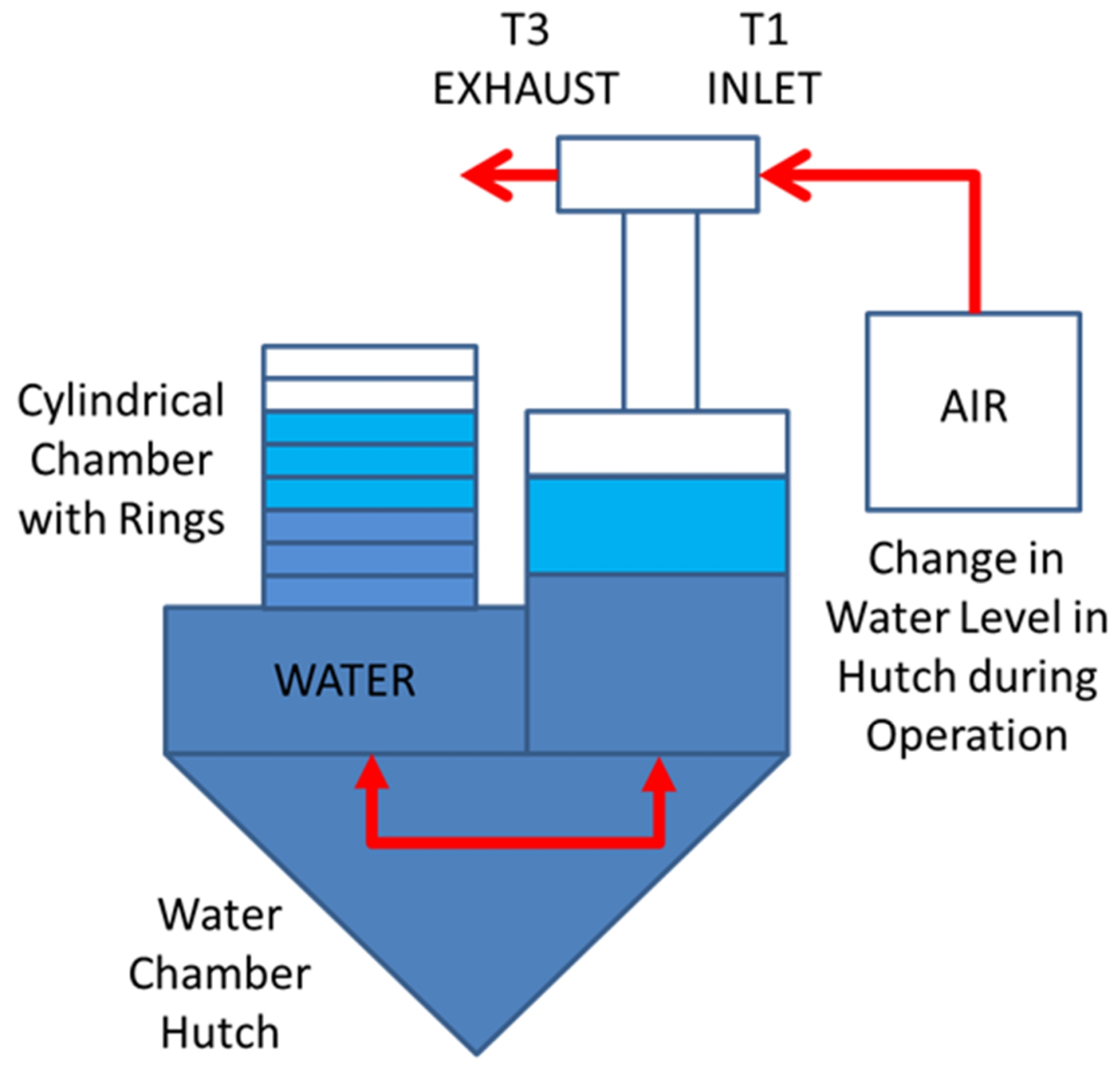

Figure 1. The Mineral Density Separator (after Van Wyk, 2006).

Batch jigging operations (as the MDS) make use of the same stratification principles experience in convention continuous jigs and can therefore be used to simulate the jigging process on a small scale. The MDS will then simulate the particle stratification movement of a jig. In a mineral jig, a deep bed of particles is supported on a horizontal perforated surface and is immersed in water (Woollacott and Eric, 1994). Jigging action causes the water level in the bed to rise and fall, so that the bed is dilated and consolidated in a cyclical fashion. The 
three principles of particle movement in jigs were defined by Gaudin (1939) as hinderedsettling, differential acceleration and consolidation trickling.

Settling phenomena, especially hindered settling, underlie all gravity concentration processes. However, most hindered settling models have concentrated on estimating hindered settling velocities based on simple suspensions consisting of either constant density distributions or uniform size distributions (Al-Naafa and Selim, 1992; Davis and Gecol, 1994). Despite various attempts to describe stratification in jigs (Zigrang and Sylvester, 1981; Mishra and Mehrotra, 1998; Richardson and Zaki,1954; Galvin et al.,1999; Lockett and Al-habboody, 1974; Garside and Al-Dibouni, 1979; Masliyah, 1979; Barnea and Misrahi, 1973; Mondal, 1997; Concha and Almendra,1979; Brauer and Theile, 1973; Asif, 1997), existing theory is not yet sufficiently well developed to provide quantitative explanations of jigging.

In this paper, the three-dimensional movement of particles of different densities and sizes inside the MDS is therefore considered based on experiments with particles colour coded for density, as well as by means of a dimensionally reduced physical model of the batch jig, which can in principle be used to track individual particles.

\section{Experimental work}

A low porosity banded iron ore sample, representing the iron ore deposit being investigated, was identified for conducting the test work. The material in the MDS was separated into layers of light, heavy and middling products, which were distributed throughout the cylindrical chamber of the MDS according to their densities. After separation, the material was removed and the bulk density was determined for each of the ten layers in the device. This bulk density showed an increase in density from the top of the MDS to the bottom. This bulk density gave an average density and did not indicate the real density composition of each layer.

Samples were classified in sink-float analyses through the use of dense medium separation with ferrosilicon and tetrabromoethane. The particles were subsequently spray painted, with each colour representing a specific density, as indicated in Table 1. Although the spray paint could influence the particles density by preventing water from filling the pores inside the particles in the jig, this effect was considered to be negligible, owing to the low porosity of the particles. The size distribution of the particles in each density interval was determined by screen analyses, ranging from $+3.2 \mathrm{~mm}$ up to $8 \mathrm{~mm}$. This size range was chosen to represent the real life jigging plant operation.

Fifteen replicate runs were conducted with pulse cycles and parameter settings as indicated in Figure 2. 
Table 1. Density intervals of iron ore sample determined by use of dense medium separation.

\begin{tabular}{|c|c|c|c|c|c|c|}
\hline Colour & White & Blue & Purple & Green & Yellow & $\begin{array}{c}\text { No } \\
\text { Colour }\end{array}$ \\
\hline $\begin{array}{c}\text { Density Range } \\
\left(\mathbf{k g} / \mathbf{m}^{\mathbf{3}}\right)\end{array}$ & $>4000$ & $3800-4000$ & $3600-3800$ & $3400-3600$ & $3200-3400$ & $<3200$ \\
\hline
\end{tabular}




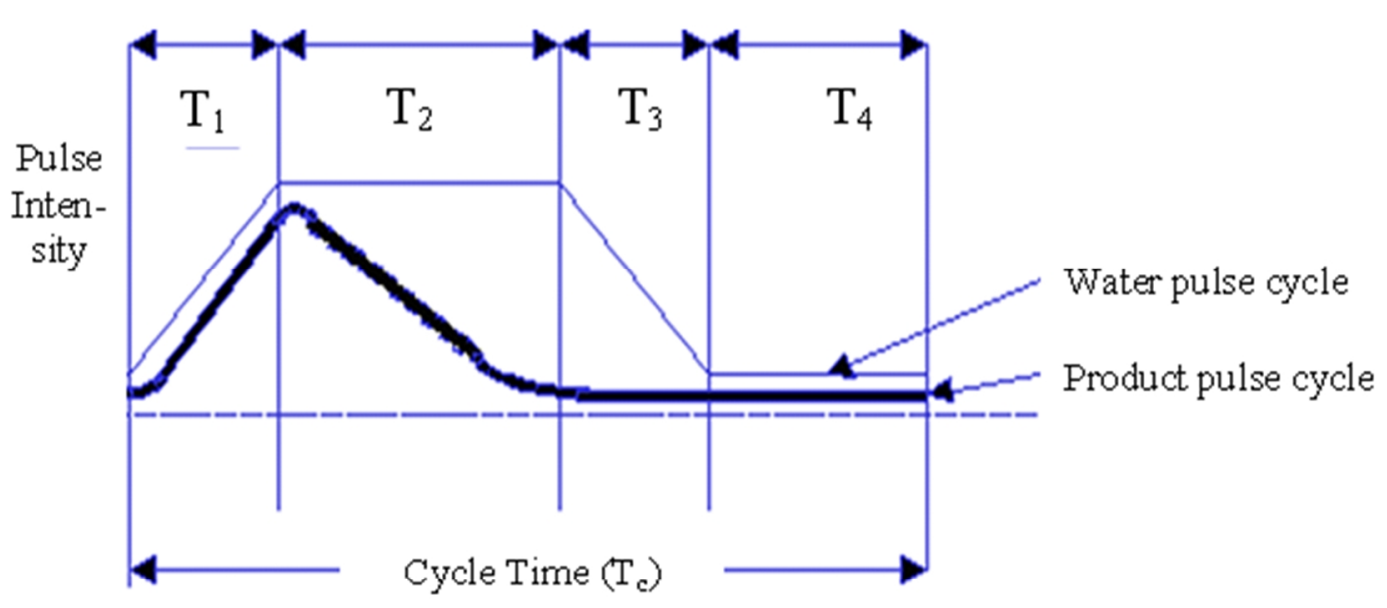

\section{Figure 2. Pulsing cycles in the batch jig.}

\section{$2.1 \quad$ Open time $\left(T_{1}\right)[180 \mathrm{~ms}]$}

At time $\mathrm{T}_{1}$ an upward pulse was exerted through the bed by blowing air into the system to displace the water that lifted the particles in the bed. With this action, some of the lighter particles were washed past the heavier particles, resulting in the formation of a loosened bed. The actual mechanism of upgrading in the bed is still not well understood. Two possible mechanisms are at work, namely diffusion (function of the local turbulence in the bed which has a remixing effect) and mobility (separation function of the density of the particle relative to the density of the surrounding environment). The dominant effect will depend on the operation of the MDS.

\section{$2.2 \quad$ Hold time $\left(T_{2}\right)[360 \mathrm{~ms}]$}

During the hold time, the bottom layer of the lifted bed (heavier particles) settled first, followed by increasingly lighter particles to generate a wave of settling that passed upwards through the lifted bed as it settled. The hold period had to be long enough to allow consolidation of the bed at the bottom of the chamber, otherwise fine and light particles could be sucked into the still expanded particle bed.

\section{$2.3 \quad$ Exhaust period $\left(T_{3}\right)[190 \mathrm{~ms}]$}

During the exhaust period the water flowed back into the water/air chamber. As with the other parts of the cycle, the setting of this period was also important. Too long an exhaust period would cause the water to overflow at the exhaust, while too short a period would force air into the system, causing bubbling, which would adversely affect the separation process. 
In addition, $T_{3}>T_{1}$, since the gravitational settling time of the particles was longer than the time required for their upward movement created by the pressure force during $T_{1}$.

The exhaust period (down time) was the return period where the air cylinder returned to the start position. Since the cylinder always had to start at the same position, a limit switch was installed. In addition, a PLC controlled valve was installed in the air line to ensure that the cylinder returned to the normal position at a slow rate.

\section{$2.4 \quad$ Runout time $\left(T_{4}\right)[280 \mathrm{~ms}]$}

The run-out time ensured that the same number of pulses were generated over a specific running time. The T4 period was adjusted to ensure completion of the desired cycle time, i.e., if the desired cycle time $\left(\mathrm{T}_{\mathrm{c}}\right)$ was $1000 \mathrm{~ms}$; with $\mathrm{T}_{1}=210 \mathrm{~ms} ; \mathrm{T}_{2}=250 \mathrm{~ms}$ and $\mathrm{T}_{3}=220 \mathrm{~ms}$, then $\mathrm{T}_{4}$ was set to $320 \mathrm{~ms}$ to complete the desired cycle time.

\subsection{Cycle time $\left(T_{c}\right)$ and residence time}

The cycle time $\left(T_{c}\right)$ was the total time of a pulse, $\left(T_{1}-T_{4}\right)$, and it was related to the frequency of pulsations. $T_{c}$ could be manipulated to achieve the required number of pulses over a given period. The longer the run-out time, the longer it took to complete a pulse, which resulted in fewer pulses per given period. This meant that a longer residence time was needed to achieve a good recovery.

The residence time (total time particles spend in MDS) was the total operational time of the MDS and had to be set so that the desired yield could be obtained at a specified grade. The MDS is a batch type operation and therefore all the particles had the same residence time. The standard residence time for the MDS was set at 30 minutes to ensure achievement of the maximum grade.

After completion of each run, the individual layers were removed from the MDS and dried. The particles in each layer were separated manually based on density (colour). Further separation of the density fractions into different size fractions was done using a standard laboratory dry screening procedure. This yielded six density fractions, each of which were separated into six different particle size fractions.

\section{Discussion of Results}

\subsection{Effect of ring size on bed lift}

The relative displacements of the water and particles are much larger when using small diameter rings than for large rings under the same conditions. It is with this in mind that times $\mathrm{T}_{1}-\mathrm{T}_{3}$ should be decreased when using small rings in order to obtain an equal relative displacement in the cylindrical chamber. Large rings $(315 \mathrm{~mm}$ diameter) should be used when large particles $(25 \mathrm{~mm})$ are concentrated and small rings (200 $\mathrm{mm}$ diameter) should be used for $8 \mathrm{~mm}$ particles. 


\subsection{Iron and silica distribution in stratified layers}

Figure 3 shows the total iron and silica distribution per layer for the samples from different test runs. As indicated in the Figure, sharp separation between the iron and the silica mostly associated with the gangue materials could be achieved between the third and the fourth rings from the bottom of the chamber.

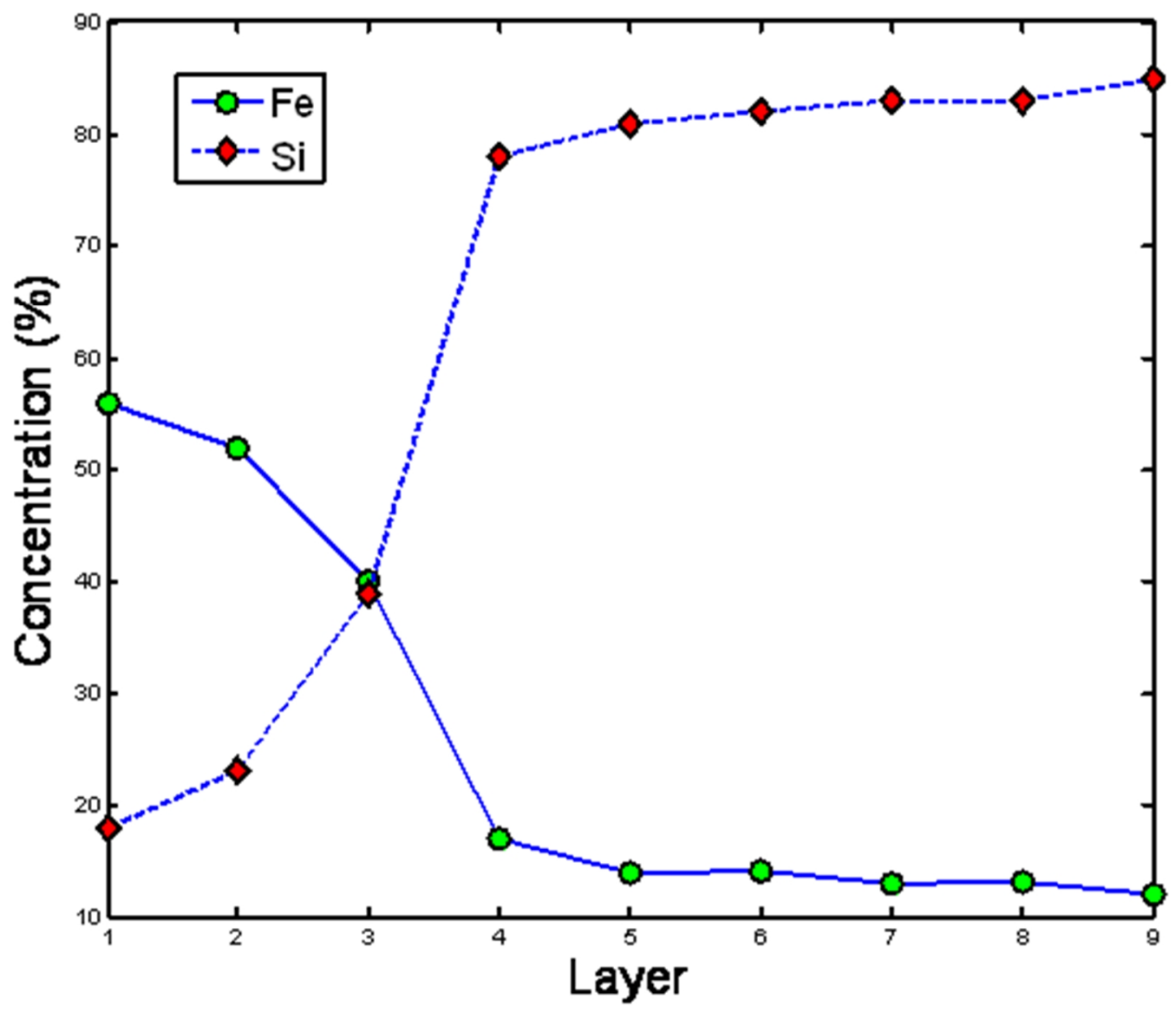

Figure 3. Typical total iron and silica distribution for test runs.

\subsection{Visual depiction of density distribution in stratified layers}




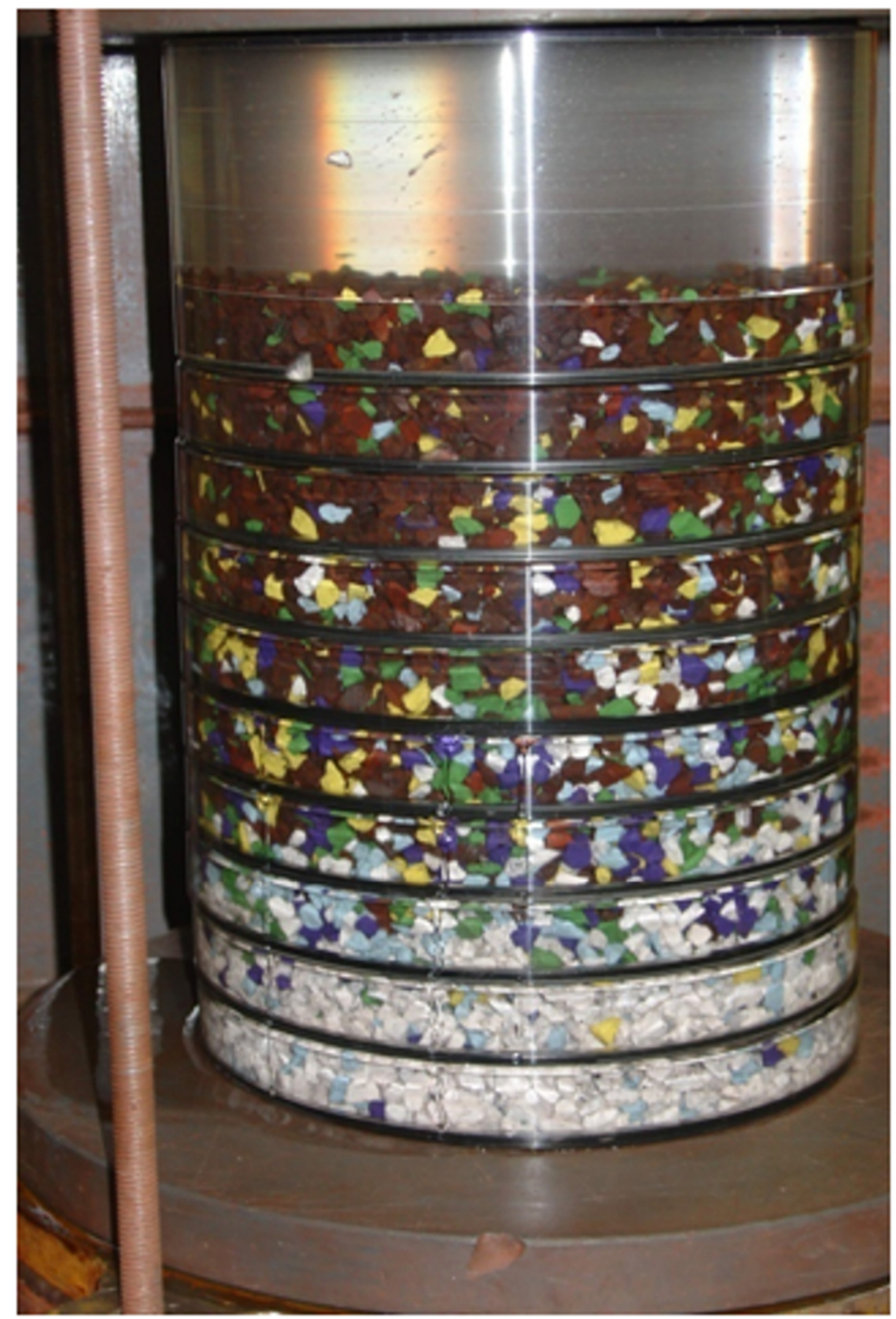

Figure 4 shows the particles in the ring chamber of the jig after separation. The stratification of the different particles based on density (colour) suggests that separation achieved in the MDS is highly efficient, as the white particles (representing most of the iron ore) are concentrated at the bottom of the device. The density of this specific banded iron ore sample is approximately $4800 \mathrm{~kg} / \mathrm{m}^{3}$. 


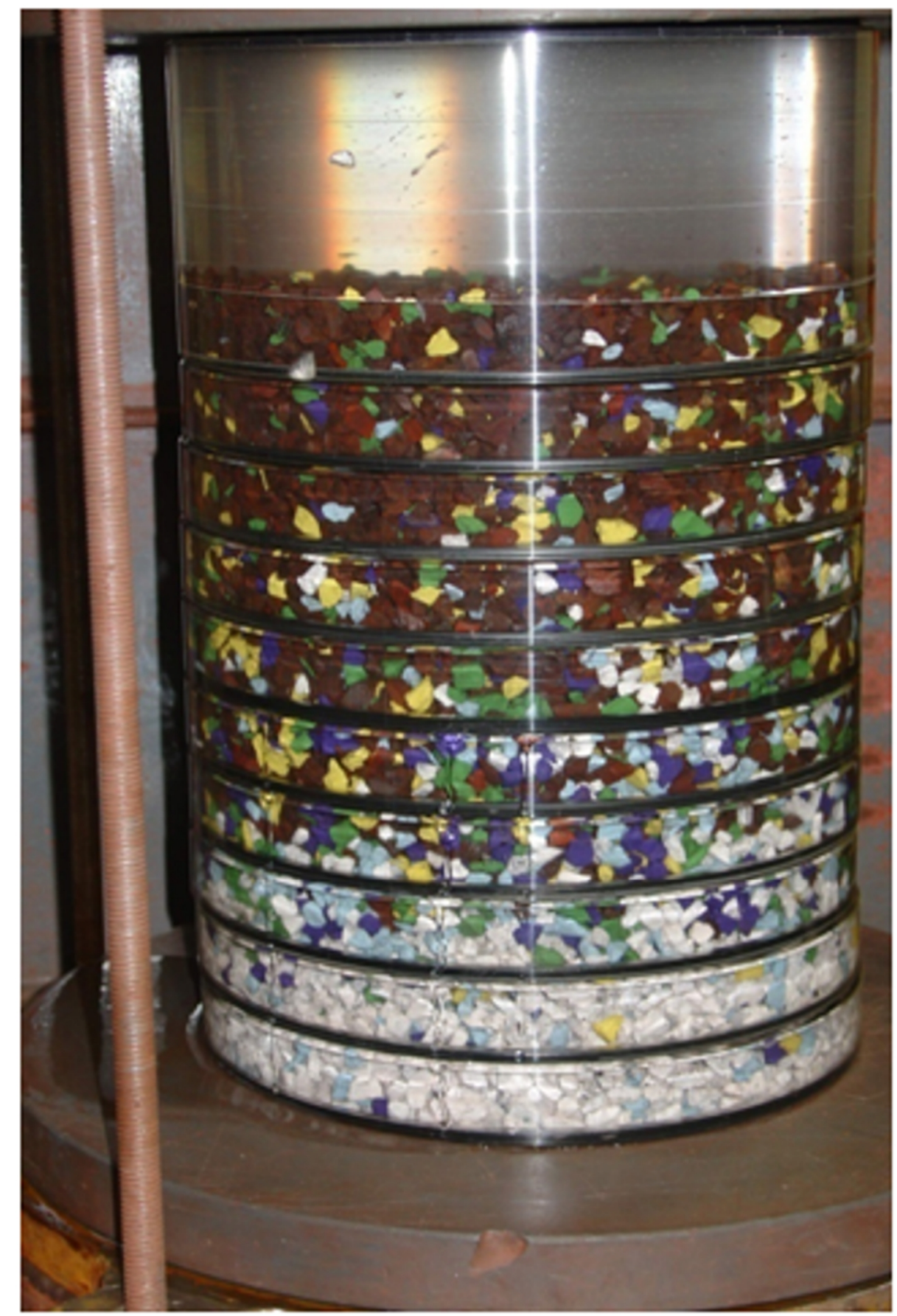

Figure 4. Stratification of the ore particles in the chamber of the jig.

The rest of the particles are all mixed together in the top part of the MDS, with no clear separation between the various densities. This is owing to the very small density differences $\left(3200 \mathrm{~kg} / \mathrm{m}^{3}\right.$ to $\left.3800 \mathrm{~kg} / \mathrm{m}^{3}\right)$ between the rest of the particles. Particles with a density lower than $3200 \mathrm{~kg} / \mathrm{m}^{3}$ (non-coloured particles), can already be found in the third layer from the bottom. This is unexpected as the fundamental theory on gravity separation indicates that these particles should have been in the top part of the unit (Kuang et al., 2008).

MDS stratification according to size $(+6.7 \mathrm{~mm})$ and density is summarized in Figure 5. Similar trends in stratification were observed for all the other size fractions investigated. 
High density particles $\left(>4000 \mathrm{~kg} / \mathrm{m}^{3}\right)$ stratification takes place in the bottom layers of the MDS (layers 1 to 4 ) and a definite separation of high density particles (white) is apparent (Figure 4). Stratification according to size is observed for the high density particles with $56.5 \%$ of particles reporting to layer 1 . For the low-density particles $\left(<3200 \mathrm{~kg} / \mathrm{m}^{3}\right)$ it would be expected that the misplacement will mainly be on the bigger sizes $(+6.7 \mathrm{~mm})$ but this size fraction showed a binomial distribution from layer 5 upwards. The effect of size on the stratification of low-density particles is not clear.

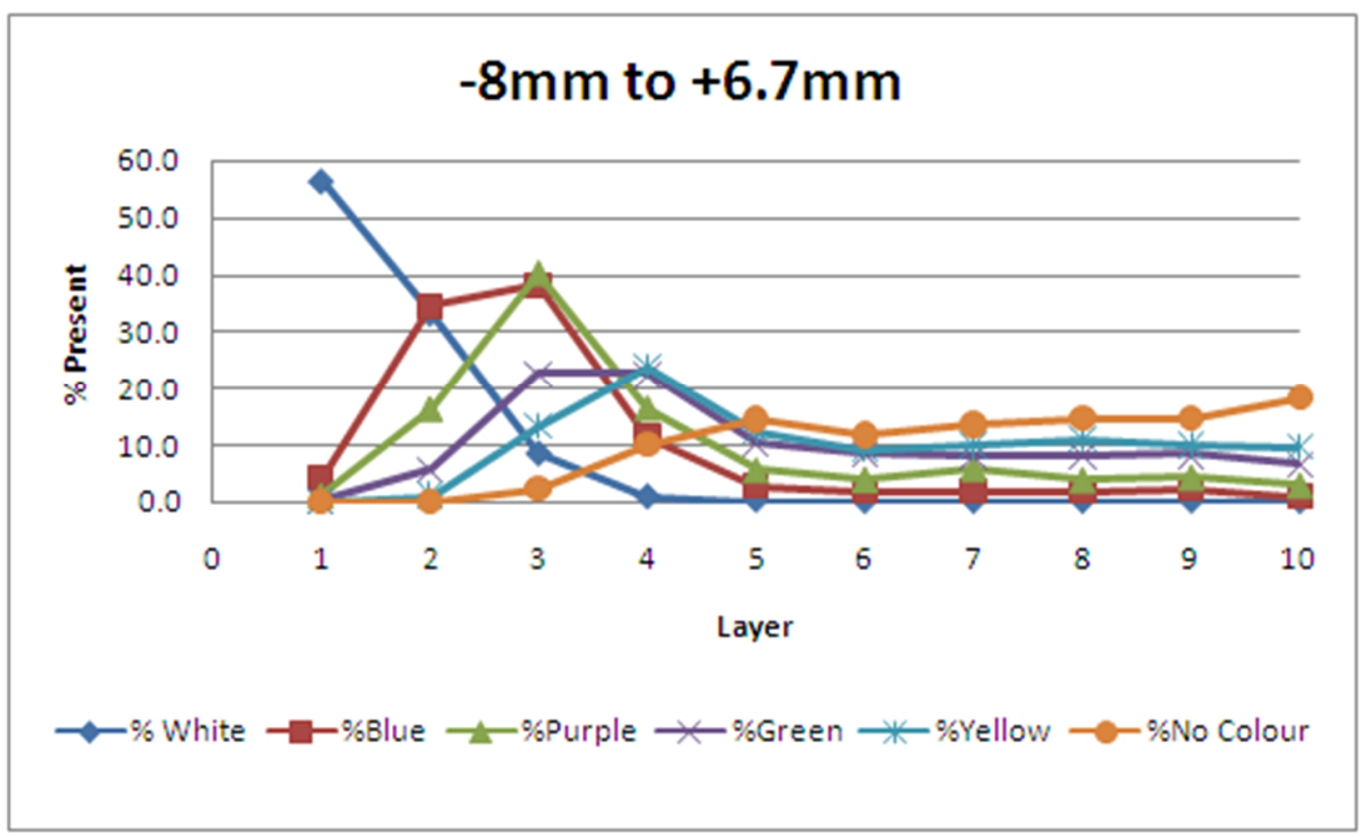

Figure 5. Size distribution per density and layer inside the MDS (White $4000 \mathrm{~kg} / \mathrm{m}^{3}$, Blue $3800 \mathrm{~kg} / \mathrm{m}^{3}$, Purple $3600 \mathrm{~kg} / \mathrm{m}^{3}$, Green $3400 \mathrm{~kg} / \mathrm{m}^{3}$, Yellow $3200 \mathrm{~kg} / \mathrm{m}^{3}$, No Colour $<3200 \mathrm{~kg} / \mathrm{m}^{3}$.

\section{Partitioning function of the MDS}

To summarize the separation efficiency of the device, a Weibull function (Rao, 2004) was fitted to the data according to eq (1). Fitting the four parameters $\left(Y_{p}, \rho_{p}, p\right.$ and $\left.q\right)$ was done with the Levenberg-Marquardt least squares algorithm in Matlab. The sum of squares and mean squares errors based on 432 samples are indicated in Figure6. The fit was slightly better than what could be obtained with a logistic function, but was not very satisfactory, as can be seen from the poor fit of the surface in the large size, high density region of the particles. Problems with convergence of the algorithm could have been a consequence of the relatively narrow size range of the particles. 


$$
Y=100\left(1-\exp \left(-\ln \left(\frac{1}{1-Y_{p}}\right)\left(\frac{\rho}{\rho_{p}}\right)^{p d^{q}}\right)\right)
$$

with $Y_{p}=-0.0442, \rho_{p}=3026.8, p=5.9475$ and $q=0.4897$

A significantly better fit could be obtained by use of a multilayer perceptron neural network with four inputs, one output and a single sigmoidal hidden layer of three nodes, trained with a second order gradient descent method. The results of the fit with the neural network are shown in Figure 7.

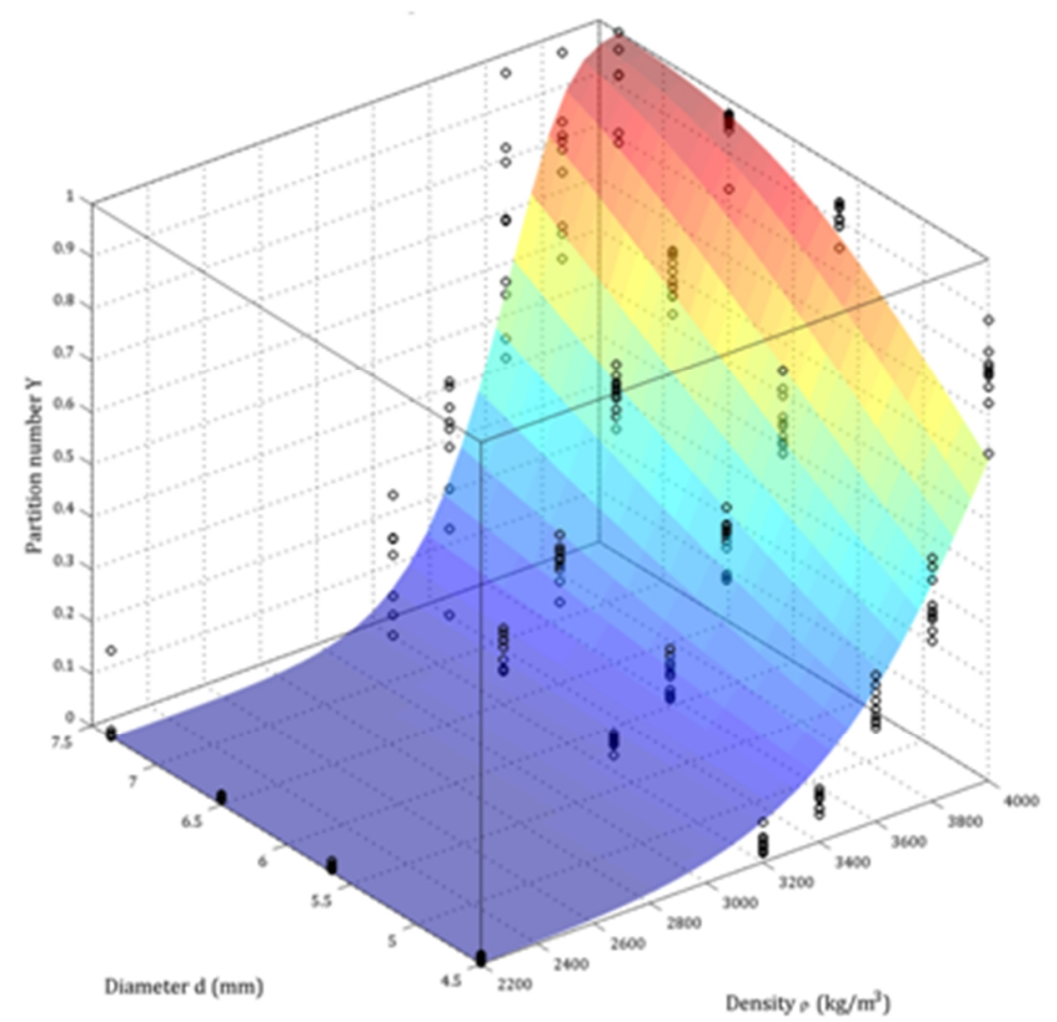

Figure 6. Partition number as a function of particle size and density according to eq. (1). 


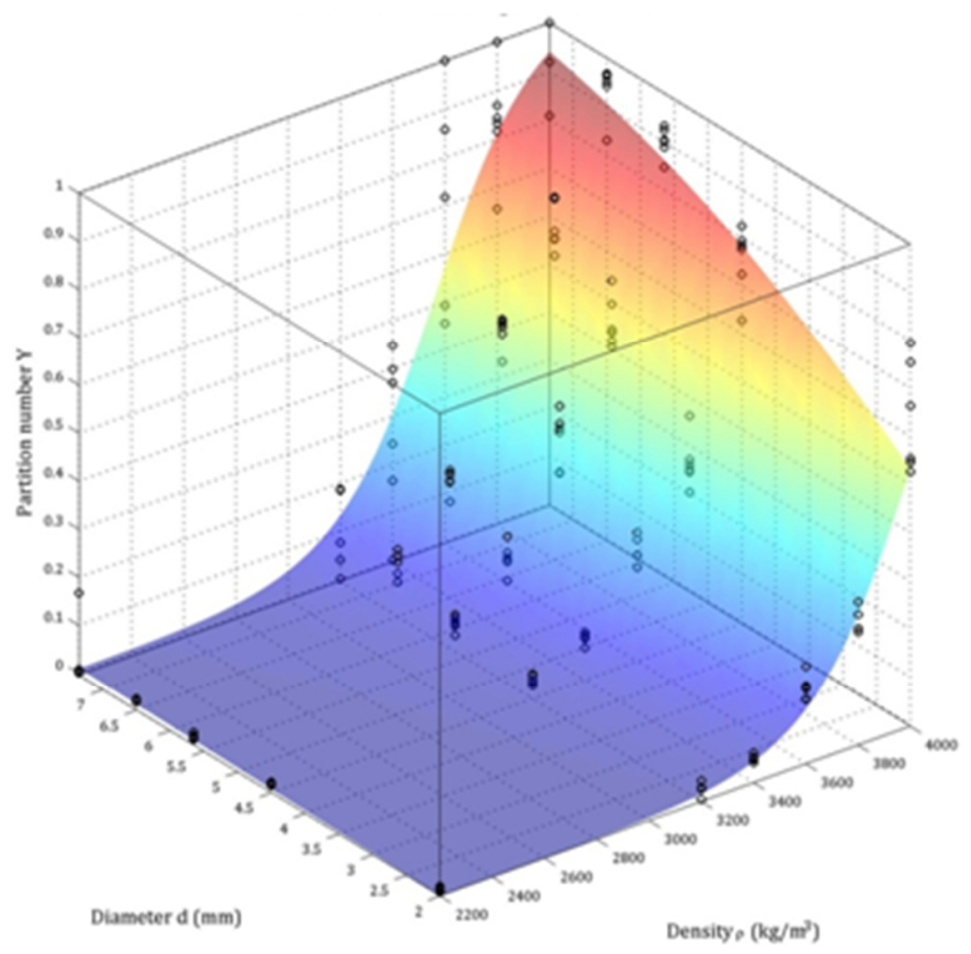

Figure 7. Partition number as a function of particle size and density fitted with a neural network.

\section{Discussion and Conclusions}

In this study, the particle density and size distributions of iron ore inside a batch jig could be traced in a series of experimental runs. Sharp separation between the high density iron ore and the gangue materials was observed, which confirmed the high separation efficiency of the jig. 
However, the least dense particles $\left(<3200 \mathrm{~kg} / \mathrm{m}^{3}\right)$ could not be stratified, despite prolonged operation of the jig. This can be seen from

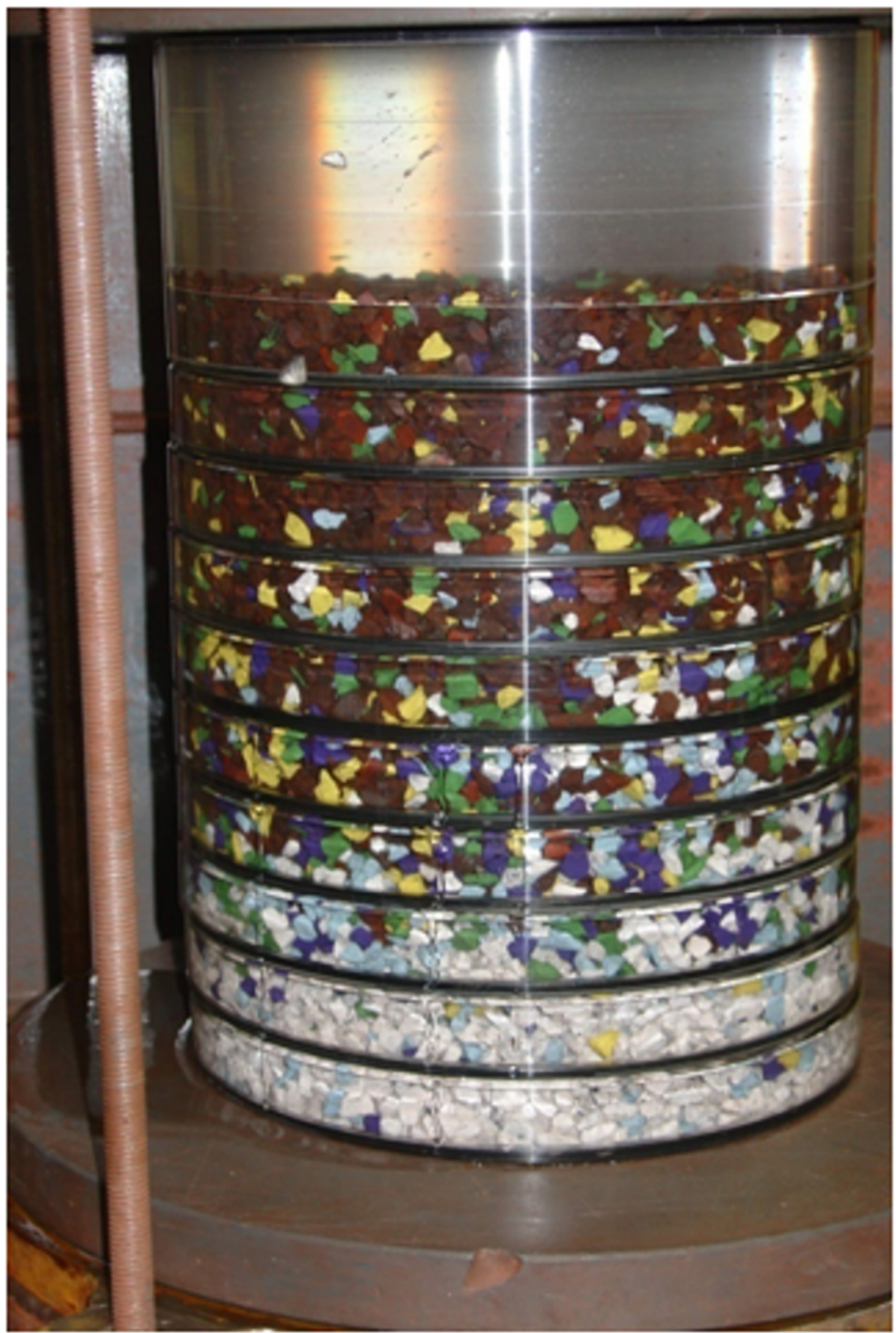

Figure 4, which shows the unpainted $\left(<3200 \mathrm{~kg} / \mathrm{m}^{3}\right)$, yellow $\left(3200-3400 \mathrm{~kg} / \mathrm{m}^{3}\right)$ and green $\left(3400-3600 \mathrm{~kg} / \mathrm{m}^{3}\right)$ particles occurring in all the rings, except the bottom two. In fact, practically no further stratification of the samples could be observed after approximately two minutes of operation of the jig. 


\section{Acknowledgements}

The authors are grateful for the contributions of Mr D van Wyk, Prof T Van Molte and Kumba Iron Ore Ltd.

\section{References}

Al-Naafa, M.A., and Selim, M.S. 1992. Sedimentation of monodisperse and bidisperse hardsphere colloidal suspension." AIChE. Journal, 38(10), 1618-1630.

Asif, M., (1997), "Modeling of multi-solid liquid fluidized-beds. Chemical Engineering Technology, 20, 485-490.

Barnea, E., and Mizrahi, J. 1973. A generalized approach to the fluid dynamics of particulate systems. Part I. Chemical Engineering Journal, 5, 171-189.

Brauer, H., and Theile, H. 1973. Bewegung von Partikelschwarmen (German). Chem. Ing. Tech., 45(13), 100-121.

Concha, F., and Almendra, E.R. 1979. Settling velocities of particulate systems. Settling of suspensions of spherical particles. International Journal of Mineral Processing, 6, 31-41.

Davis, R.H., and Gecol, H. 1994. Hindered-settling function with no empirical parameters for polydisperse suspension. AIChE Journal, 40(3), 570-575.

Galvin, K.P., Prattern, S.J., and Nguyen-Tran-Lam, G. 1999. A generalized empirical description for particle slip velocities in liquid fluidized-beds. Chemical Engineering Science, $54,1045-1052$.

Garside, J., and Al-Dibouni, M.R. 1979. Particle mixing and classification in liquid fluidizedbeds. Transactions of the Institute of Chemical Engineering, 57, 95-103.

Gaudin, A. M. 1939. Principles of Mineral Dressing. McGraw-Hill, New York, NY, USA.

Kuang, U., Zhuo, J., Wang, L. and Yang, C. 2008. Laws of motion of particles in a jigging process. Journal of China University of Mining and Technology, 18(4), 575-579.

Lockett, M.J., and Al-Habboody, H.M. 1974. Differential settling by size of two particle species in a liquid. Transactions of the Institute of Chemical Engineering, 52, 281-292.

Masliyah, J.H. 1979. Hindered-settling in a multi-species particle system. Chemical Engineering Science, 34, 1166-1168.

Mishra, B.K., and Mehrotra, S.P. 1998. Modeling of particle stratification in jigs by the discrete element method. Minerals Engineering, 11(6), 511-522. 
Mondal, K. 1997. A dynamic population balance model for a hindered bed classifier for fine particle separations. Ph.D. Thesis, Southern Illinois University, Carbondale, IL, USA.

Rao, B.V. 2004. Weibull partition surface representation for gravity concentrators. Minerals Engineering, 17, 953-956.

Richarson, J., and Zaki, W. 1954. Sedimentation and fluidization: Part I. Transactions of the Institute of Chemical Engineering, 39, 35-53.

Woollacott, L.C., and Eric, R.H. 1994. Mineral and Metal Extraction and overview. Southern African Institute of Mining and Metallurgy, Johannesburg. South Africa. 140 \& 164.

Zigrang, D.J., and Sylvester, N.D. 1981. An explicit equation for particle settling velocities in solid-liquid systems. AIChE Journal, 27(6), 1043-1044. 